\title{
Persistent Enlarged Occipital Sinus with Absent Unilateral Transverse Sinus
}

\author{
Guru Dutta Satyarthee ${ }^{1}$ Luis Rafael Moscote-Salazar² \\ ${ }^{1}$ Department of Neurosurgery, Neurosciences Centre, All India \\ Institute of Medical Sciences, New Delhi \\ 2Department of Neurosurgery and critical care, RED LATINO, Latin \\ American Trauma and Intensive Neuro-Care Organization, Bogota, \\ Colombia \\ ${ }^{3}$ Department of Neurosurgery, Narayana Medical College Hospital, \\ Nellore, Andhra Pradesh, India
}

\author{
Amit Agrawal $^{3}$
}

J Neurosci Rural Pract 2019;10:519-521

\begin{abstract}
Keywords

- absent transverse sinus

- acoustic schwannoma

- patent occipital sinus

- posterior fossa

- surgical exposure
\end{abstract}

\begin{abstract}
Address for correspondence Guru Dutta Satyarthee, MCh, Department of Neurosurgery, Room No. 714, Neurosciences Centre, All India Institute of Medical Sciences, New Delhi, India (e-mail: duttaguru2002@yahoo.com).
\end{abstract}

\section{Introduction}

Usually, patent occipital sinus (POS) is observed in $<10 \%$ of human population. A persistent POS associated with absent transverse sinus is extremely uncommon occurrence and not reported. The neurosurgeon and otolaryngo-rhinologist should be aware about the possibility of associated venous sinus anomaly as it can affect surgical planning, selection of appropriate approaches, and may critically limit the surgical exposure of target. As routinely magnetic resonance (MR) venography is not routinely utilized for assessing the anatomy of venous sinus of posterior fossa lesion including cerebellopontine angle and cerebellar neoplasms, but authors advocate MR venography in addition to MR imaging (MRI) study should be made an integral part of neuroimaging study.

\section{Case Report}

A 35-year-old male patient presented with diminution of hearing on right side for 2 years and imaging revealed right-sided giant acoustic schwannoma. He was taken up for surgical excision after detailed prognostication and obtaining consent under general anesthesia in lateral position. After raising scalp flap and elevating bone flap, prominent occipital sinus was observed, which was not placed exactly in the midline but toward right side, associated with absent left side transverse and sigmoid sinus ( - Fig. 1). The persistence occipital sinus caused limited availability of the overall exposed dural surface for intended durotomy and surgical exposure of schwannoma. The critically limited surgical exposure led to struggle and prolonged operative time with associated 


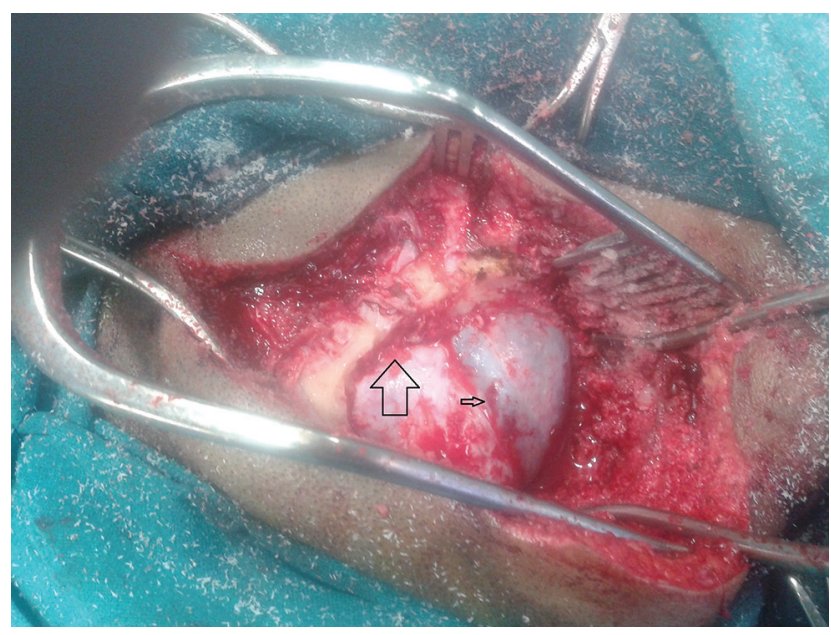

Fig. 1 Intraoperative photograph showing persistent dilated, tortuous, occipital sinus (arrow head) with absent transverse sinus (arrow) led to severe restriction of dural opening and grossly minimizing surgical exposure.

increased blood loss and difficulty encountered while preserving the neurovascular structure.

However, surgical ligation of the occipital sinus was not contemplated, in view of associated absent contralateral absent transverse sinus, in view of obliteration of venous return and brain bulge. Microscopic gross total surgical resection with anatomical preservation of facial nerve was performed. The postoperative period was uneventful. A reaper MRI brain with venography was planned in the immediate postoperative period; however, due to very poor economic status, he showed inability and so planned for interval MR venography during follow-up period, so that we can be able to judge the extent of surgical resection and look for any residual as well as patency of occipital sinus.

\section{Discussion}

The occipital sinus connects torcular to the marginal sinus. Occipital sinuses are considered as the smallest caliber among the all cerebral dural venous sinuses. Persistence occipital sinus is usually associated with absence or hypoplastic unilateral transverse sinus as well as sigmoid sinuses. ${ }^{1,2}$ It is commonly observed relatively in the neonate, but gradually disappears and may be present in approximately $10 \%$ of the normal population. Persistence of POS has important surgical significance in the selection of various surgical approaches for targeting lesions located in the posterior fossa including retrosigmoid approaches..$^{13-5}$

Embryologically, the occipital sinus originates as small venous channels usually originating from the primitive torcula and medial portions of transverse sinuses and most prominent during the fifth month of intrauterine life and shows a progressive reduction in caliber and number and finally at birth only seen in minority of the population. Occipital sinuses may not be always located in the midline and may show variable course. ${ }^{1,2}$ It usually courses inferiorly from torcula toward the marginal sinus located around foramen magnum and finally, in turn, drains into suboccipital veins, paravertebral venous plexus, or jugular veins. ${ }^{1}$ Manjunath observed that venous dural sinuses anomaly can have diverse implication including radiological finding, surgical planning, and limited surgical exposure and difficulty in the execution of the surgical procedure. ${ }^{2}$

Manjunath analyzed a total of 214 sides of the skull bases and finally concluded that 10 sides had demonstrable presence of anomaly of the dural sinus, but out of them, only one case was associated with the bilateral absent groove for transverse and sigmoid sinuses but rest had unilateral absence of transverse sinus groove. ${ }^{2}$ Tada et al reported a pediatric case with a diagnosis of neck swelling associated with intracranial sinus abnormality involving persistent enlarged occipital sinus and relatively much-narrowed caliber of the transverse sinus. $^{6}$

It is imperative that, when planning posterior fossa surgery including retrosigmoid surgical approach, the anatomy of venous sinus should also be considered as part of minimum imaging workup, including MR venography to a void last-minute misadventure, intraoperatively after craniectomy. ${ }^{7}$ Further, if POS detected during any radiological study, it needs to be specially taken into account for surgical planning, and associated anomaly must be documented, and neurosurgical team must develop microsurgical technique in neurosurgical armamentarium to deal effectively and successfully without compromising patient safety and without aggravating neurological morbidity.

\section{Conclusion}

A persistent POS represents uncommon venous anomaly of venous sinus of posterior fossa and further may be associated with other venous sinus abnormality of the posterior cranial fossa. Absent transverse sinus with POS is an extremely uncommon occurrence but represent the important clinical entity and neurosurgical team. Neuroradiologist and otolaryngologist must be aware that POS with absent transverse and sigmoid sinus may necessitate surgical planning modification, appropriate microneurosurgical approaches selection, and additionally may limit the extent of surgical exposure for further emphasis of detailed neuroanatomy. Finally, neuroimaging is justified to avoid intraoperative chaos.

\section{Funding \\ None.}

\section{Conflict of Interest}

None declared.

\section{References}

1 Widjaja E, Griffiths PD. Intracranial MR venography in children: normal anatomy and variations. AJNR Am J Neuroradiol 2004;25(9):1557-1562 
2 Manjunath KY. Anomalies of transverse and sigmoid sinuses associated with contracted jugular foramina. Indian J Otolaryngol Head Neck Surg 2004;56(2):108-114

3 Satyarthee GD. Predicting shape, location, and course of facial nerve in relation to large vestibular schwannoma on diffusion tensor imaging with intraoperative correlation: Important surgical adjunct. World Neurosurg 2017; 105:1002

4 Satyarthee GD, Jain G. Indication of surgical management of vestibular schwannoma in the older age group: mysteries still unsolved. World Neurosurg 2017;101:803-804
5 Satyarthee GD, Kumar S. Is staged surgery for giant vestibular schwannomas always better in improving outcome: needs socioeconomic consideration? J Neurosci Rural Pract 2014;5(4):437-439

6 Tada H, Takanashi J, Barkovich AJ, Kohno Y. Intracranial dural venous anomalies in familial cervical cystic hygroma. Pediatr Neurol 2005;32(1):50-52

7 Salem M, Dolati P, Fusco MR, Ogilvy CS, Thomas AJ. Abnormal large central occipital emissary vein: a case report and review of literature. Cureus 2016;8(5):e603 